PROCEEDINGS OF THE

AMERICAN MATHEMATICAL SOCIETY

Volume 133, Number 5, Pages 1291-1298

S 0002-9939(04)07680-4

Article electronically published on October 18, 2004

\title{
WEAK BOUNDEDNESS THEOREMS FOR CANONICALLY FIBERED GORENSTEIN MINIMAL 3-FOLDS
}

\author{
MENG CHEN \\ (Communicated by Michael Stillman)
}

\begin{abstract}
Let $X$ be a Gorenstein minimal projective 3-fold with at worst locally factorial terminal singularities. Suppose the canonical map is of fiber type. Denote by $F$ a smooth model of a generic irreducible element in fibers of $\phi_{1}$, and so $F$ is a curve or a smooth surface. The main result is that there is a computable constant $K$ independent of $X$ such that $g(F) \leq 647$ or $p_{g}(F) \leq 38$ whenever $p_{g}(X) \geq K$.
\end{abstract}

\section{INTRODUCTION}

In this paper, we study the boundedness problem of a canonical family of curves or surfaces on certain algebraic 3-folds. Let $X$ be a Gorenstein minimal projective 3 -fold with locally factorial terminal singularities. Suppose $p_{g}(X) \geq 2$. One may define the canonical map $\phi_{1}$. Denote $\alpha:=\operatorname{dim} \phi_{1}(X)$. We say that $\phi_{1}$ is of fiber type if $\alpha<3$. Denote by $F$ a smooth model of a generic irreducible element in fibers of $\phi_{1}$. It is interesting to know whether the birational invariants of $F$ are bounded. For technical reasons, we have to assume on $X$ an effective Miyaoka-Yau inequality. This is the reason that we are only able to treat a Gorenstein minimal 3 -fold by virtue of ([14]). Apparently we have $\kappa(X) \geq 1$. If $\kappa(X) \leq 2$, by the Base Point Free Theorem ([11, 13), one can see that $F$ is special. So, throughout, we may suppose $\kappa(X)=3$, i.e. $X$ is of general type. What we can do is as follows (see Theorem 1.5, Theorem 2.3 and Theorem 2.8).

Theorem 0.1. There is a computable constant K. For all $X$, a Gorenstein minimal projective 3 -fold with locally factorial terminal singularities, denote by $\phi_{1}$ the canonical map of $X$ and by $F$ a smooth model of a generic irreducible element in fibers of $\phi_{1}$. The following hold:

(1) If $\operatorname{dim} \phi_{1}(X)=2$, then $F$ is a curve with geometric genus $g(F) \leq 647$ provided $p_{g}(X) \geq K$.

(2) If $\operatorname{dim} \phi_{1}(X)=1$, then $F$ is a surface of general type with geometric genus $p_{g}(F) \leq 38$ provided $p_{g}(X) \geq K$.

Remark 0.2. In the above theorem, we do not know the boundedness when $p_{g}(X)<$ $K$. The relations among $p_{g}(X), q(X)$ and $h^{2}\left(\mathcal{O}_{X}\right)$ are expected to be known to

Received by the editors September 26, 2002 and, in revised form, January 8, 2004.

2000 Mathematics Subject Classification. Primary 14C20, 14E35.

This paper was supported by the National Natural Science Foundation of China (No.10131010), Shanghai Scientific \& Technical Commission (Grant 01QA14042) and SRF for ROCS, SEM. 
solve the problem. We also believe that those bounds in 0.1 might be far from sharp.

I thank a referee for effective suggestions. This paper was finally revised while I was visiting Kang Zuo at the Chinese University of Hong Kong. Special thanks are due to Kang Zuo for much help and hospitality.

\section{Canonical family of Surfaces}

1.1. Setting up. Let $X$ be a minimal projective 3 -fold with $\mathbb{Q}$-factorial terminal singularities. Suppose $p_{g}(X) \geq 2$. We can define the canonical map $\phi_{1}$, which is usually a rational map. Take the birational modification $\pi: X^{\prime} \longrightarrow X$, according to Hironaka, such that

(i) $X^{\prime}$ is smooth;

(ii) the movable part of $\left|K_{X^{\prime}}\right|$ is base point free;

(iii) certain $\pi^{*}\left(K_{X}\right)$ have supports with only normal crossings.

Denote by $g$ the composition $\phi_{1} \circ \pi$. So $g: X^{\prime} \longrightarrow W^{\prime} \subseteq \mathbb{P}^{p_{g}(X)-1}$ is a morphism.

Let $g: X^{\prime} \stackrel{f}{\longrightarrow} W \stackrel{s}{\longrightarrow} W^{\prime}$ be the Stein factorization of $g$. We can write

$$
K_{X^{\prime}}=\pi^{*}\left(K_{X}\right)+E=S_{1}+Z_{1},
$$

where $S_{1}$ is the movable part of $\left|K_{X^{\prime}}\right|, Z_{1}$ the fixed part and $E$ is an effective $\mathbb{Q}$-divisor that is a sum of distinct exceptional divisors.

If $\operatorname{dim} \phi_{1}(X)<3, f$ is a called a derived fibration of $\phi_{1}$. We mean a generic irreducible element in fibers of $\phi_{1}$ by a general fiber of $f$.

If $\operatorname{dim} \phi_{1}(X)=2$, a general fiber $C$ of $f$ is a smooth curve of genus $g(F)$ which should not be confused with the morphism $g$.

If $\operatorname{dim} \phi_{1}(X)=1$, a general fiber $F$ of $f$ is a smooth projective surface of general type. Denote by $F_{0}$ the minimal model of $F$. Denote by $b$ the genus of the smooth curve $W$.

Proposition 1.2. Keep the above notation. Suppose $X$ is Gorenstein minimal of general type with locally factorial terminal singularities, $\operatorname{dim} \phi_{1}(X)=1$ and $b=0$. If $p_{g}(X) \geq 2 k+2$ for certain $k \geq 4$, then there is an effective $\mathbb{Q}$-divisor $E_{k}^{\prime}$ on $X^{\prime}$ such that

$$
\left.\pi^{*}\left(K_{X}\right)\right|_{F}=\mathbb{Q} \frac{k}{k+1} \sigma^{*}\left(K_{F_{0}}\right)+E_{k}^{\prime}
$$

where $\sigma: F \rightarrow F_{0}$ is contraction onto the minimal model.

Proof. Let $M_{k+1}$ be the movable part of $\left|(k+1) K_{X^{\prime}}\right|$. Then we can write

$$
(k+1) \pi^{*}\left(K_{X}\right) \sim M_{k+1}+E_{k+1}^{\prime}
$$

where $E_{k+1}^{\prime}$ is an effective divisor with respect to $k$. Therefore we see that $\left.(k+1) \pi^{*}\left(K_{X}\right)\right|_{F} \geq\left. M_{k+1}\right|_{F}$. Let $N_{k}$ be the movable part of $\left|k K_{F}\right|$. According to [3], $\left|k K_{F_{0}}\right|$ is base point free. Thus $N_{k}=\sigma^{*}\left(k K_{F_{0}}\right)$ where $\sigma: F \longrightarrow F_{0}$ is contraction onto the minimal model.

We use the approach in [12] (Corollary 4.8) to prove $\left.M_{k+1}\right|_{F} \geq N_{k}$. In fact, we have a fibration $f: X^{\prime} \longrightarrow \mathbb{P}^{1}$. Because $p_{g}(X) \geq 2 k+2$, we can see that $\mathcal{O}(2 k+1) \hookrightarrow f_{*} \omega_{X^{\prime}}$. Thus we have

$$
\mathcal{E}:=\mathcal{O}(1) \otimes f_{*} \omega_{X^{\prime} / \mathbb{P}^{1}}^{k}=\mathcal{O}(2 k+1) \otimes f_{*} \omega_{X^{\prime}}^{k} \hookrightarrow f_{*} \omega_{X^{\prime}}^{k+1} .
$$


Note that $H^{0}\left(\mathbb{P}^{1}, f_{*} \omega_{X^{\prime}}^{k+1}\right) \cong H^{0}\left(X^{\prime}, \omega_{X^{\prime}}^{k+1}\right)$. It is well known that $\mathcal{E}$ is generated by global sections and that $f_{*} \omega_{X^{\prime} / \mathbb{P}^{1}}^{k}$ is a sum of line bundles with non-negative degree ([8, 16, 17]). Thus the global sections of $\mathcal{E}$ can distinguish different fibers of $f$. On the other hand, the local sections of $f_{*} \omega_{X^{\prime}}^{k}$ give the $k$-canonical map of $F$, and these local sections can be extended to global sections of $\mathcal{E}$. This means $\left.M_{k+1}\right|_{F} \geq N_{k}$. The proposition is proved.

Theorem 1.3 ([4], Theorem 1). Let $X$ be a minimal projective 3 -fold of general type with at worst $\mathbb{Q}$-factorial terminal singularities. Suppose $\operatorname{dim} \phi_{1}(X)=1$. If $b \geq 2$, then either

$$
\begin{gathered}
p_{g}(F)=1 \text { and } p_{g}(X) \geq b-1 \text { or } \\
b=p_{g}(F)=p_{g}(X)=2 .
\end{gathered}
$$

1.4. Reduction to the case $b=0$. We mainly study a Gorenstein minimal 3-fold. By virtue of 1.3 , one only has to consider the case $b \leq 1$. If $b=1$, then $p_{g}(F) \leq 38$ according to [4] (Theorem 2(1)). From now on within this section, we may suppose $b=0$. So $W=\mathbb{P}^{1}$.

Theorem 1.5. There exists a constant $K_{1} \leq 800$ such that, for all $X$, a Gorenstein minimal projective 3 -fold of general type with at worst locally factorial terminal singularities, satisfying $\operatorname{dim} \phi_{1}(X)=1$, the following hold:

(1) $p_{g}(F) \leq 38$ provided $p_{g}(X) \geq K_{1}$.

(2) $p_{g}(F) \leq 5002$ provided $p_{g}(X) \geq 23$.

Proof. We only have to prove the theorem for the case $b=0$. In this case, $W=\mathbb{P}^{1}$. We have a derived fibration $f: X^{\prime} \longrightarrow \mathbb{P}^{1}$. We have

$$
q(X)=h^{1}\left(\mathbb{P}^{1}, R^{1} f_{*} \omega_{X^{\prime}}\right) \leq q(F)
$$

Because $F$ is of general type, we have $p_{g}(F) \geq 2 q(F)-4$ according to [2]. Thus the Miyaoka-Yau inequality (14]) becomes

$$
\begin{aligned}
K_{X}^{3} & \leq-72\left[1-q(X)+h^{2}\left(\mathcal{O}_{X}\right)-p_{g}(X)\right] \\
& \leq 72\left[\left(p_{g}(X)-1\right)+q(F)\right] \\
& \leq 72\left[p_{g}(X)-1\right]+36 p_{g}(F)+144 .
\end{aligned}
$$

When $p_{g}(X) \geq 2 k+2$ for $k \geq 4$, Proposition 1.2 gives

$$
K_{X}^{3} \geq \frac{k^{2}}{(k+1)^{2}} \cdot K_{F_{0}}^{2} \cdot\left[p_{g}(X)-1\right] .
$$

Denote $A:=p_{g}(X)-1$ and $B:=\frac{k^{2}}{(k+1)^{2}}$. Also using the inequality $K_{F_{0}}^{2} \geq 2 p_{g}(F)-4$, we obtain the following inequality:

$$
p_{g}(F) \leq \frac{36 A+2 A B+72}{A B-18}=\frac{(36+2 B)+\frac{72}{A}}{B-\frac{18}{A}} .
$$

Noting that $A \rightarrow+\infty$ and $B \rightarrow 1$ if $k \rightarrow+\infty$, we can easily find a constant $K_{1}$ such that $p_{g}(F) \leq 38$ whenever $p_{g}(X) \geq K_{1}$. The proof is completed.

Remark 1.6. Among known examples, the largest possible $p_{g}(F)$ is 5 ([7], Example $2(\mathrm{e})$ ) where the total space is a smooth minimal 3 -fold and the canonical system is composed of a pencil of surfaces over a rational curve. Can one construct more examples with larger $p_{g}(F)$ ? 


\section{CANONicAl FAmily of CURVES}

2.1. Setting up. Within this section, we keep the same notation as in 1.1 and suppose $X$ is a Gorenstein minimal 3 -fold of general type with at worst locally factorial terminal singularities and $\operatorname{dim} \phi_{1}(X)=2$. In this case, we may even suppose $W$ is a smooth surface, and so a general fiber $C$ of $f$ is a smooth projective curve.

2.2. An inequality. We need a nontrivial inequality in terms of $g(C)$, namely,

$$
K_{X}^{3} \geq \frac{2}{3}(g(C)-1)\left(p_{g}(X)-2\right)
$$

according to 6] (Theorem 4.1(ii)). We omit the proof here simply because, on the one hand, this type of inequality is expected and, on the other hand, the proof there is quite clear and short.

Theorem 2.3. There is a computable constant $K_{2}$ such that for all $X$, a Gorenstein minimal projective 3-fold of general type with at worst locally factorial terminal singularities, satisfying $\operatorname{dim} \phi_{1}(X)=2$ and $\kappa(W) \geq 0$, the following hold:

(1) $g(C) \leq 109$ provided $p_{g}(X) \geq K_{2}$.

(2) $g(C) \leq 11989$ provided $p_{g}(X)>109$.

Proof. We have a canonically derived fibration $f: X^{\prime} \longrightarrow W$. According to 12 and [16, 17, we know that $R^{1} f_{*} \omega_{X^{\prime}} \cong \omega_{W}$ and $f_{*} \omega_{X^{\prime} / W}$ is torsion free. We have

$$
\begin{gathered}
q(X)=h^{1}\left(W, R^{1} f_{*} \omega_{X^{\prime}}\right)+h^{2}\left(W, f_{*} \omega_{X^{\prime}}\right) \\
=q(W)+h^{2}\left(W, f_{*} \omega_{X^{\prime}}\right) \leq q(W)+g(C), \\
h^{2}\left(\mathcal{O}_{X}\right)=h^{0}\left(W, R^{1} f_{*} \omega_{X^{\prime}}\right)+h^{1}\left(W, f_{*} \omega_{X^{\prime}}\right) \\
=p_{g}(W)+h^{1}\left(W, f_{*} \omega_{X^{\prime}}\right) .
\end{gathered}
$$

On the surface $W$, we have $q(W)-p_{g}(W)=1-\chi\left(\mathcal{O}_{W}\right)$. By virtue of the birational classification theory on surfaces, we can see that

$$
\chi\left(\mathcal{O}_{W}\right) \geq 0 \text { whenever } \kappa(W) \geq 0 .
$$

Thus we definitely have $q(W)-p_{g}(W) \leq 1$ under the assumption of the theorem. So we get

$$
\begin{gathered}
q(X)-h^{2}\left(\mathcal{O}_{X}\right) \leq q(W)-p_{g}(W)+g(C) \leq g(C)+1, \\
\chi\left(\omega_{X}\right)=p_{g}(X)+q(X)-h^{2}\left(\mathcal{O}_{X}\right)-1 \leq p_{g}(X)+g(C) .
\end{gathered}
$$

By 2.2 and Miyaoka's inequality, we have

$$
\frac{2}{3}(g(C)-1)\left[p_{g}(X)-2\right] \leq 72\left[p_{g}(X)+g(C)\right] .
$$

Denote $A:=p_{g}(X)-1$. The above inequality becomes

$$
g(C) \leq \frac{109 A+108}{A-108}=\frac{109+\frac{108}{A}}{1-\frac{108}{A}} .
$$

It is easy to find a constant $K_{2}$ such that $g(C) \leq 109$ provided $p_{g}(X) \geq K_{2}$.

2.4. The case $\kappa(W)=-\infty$. We know that $W$ is birationally equivalent to a ruled surface or $\mathbb{P}^{2}$. Denote $b_{0}:=q(W)$. This situation is more complicated. We have to do some preparation in order to prove the boundedness. 
Corollary 2.5. There exists a computable constant $K_{3}$ such that for all $X, a$ Gorenstein minimal projective 3-fold of general type with at worst locally factorial terminal singularities, satisfying $\operatorname{dim} \phi_{1}(X)=2$ and $\kappa(W)=-\infty$, we have $g(C) \leq$ $b_{0}+109$ provided $p_{g}(X) \geq K_{3}$.

Proof. According to the proof of 2.3, we only have to estimate $\chi\left(\omega_{X}\right)$. Because $q(X) \leq b_{0}+g(C)$, we have

$$
\chi\left(\omega_{X}\right)=p_{g}(X)+q(X)-h^{2}\left(\mathcal{O}_{X}\right)-1 \leq p_{g}(X)+b_{0}+g(C)-1 .
$$

By 2.2 and Miyaoka's inequality, we have

$$
\frac{2}{3}(g(C)-1)\left[p_{g}(X)-2\right] \leq 72\left[p_{g}(X)+b_{0}+g(C)-1\right] .
$$

Denote $A_{1}=p_{g}(X)-2$. The inequality is

$$
g(C) \leq 109 \cdot \frac{A_{1}}{A_{1}-108}+b_{0} \cdot \frac{108}{A_{1}-108}+\frac{108}{A_{1}-108} .
$$

Noting that

$$
\frac{A_{1}}{A_{1}-108} \mapsto 1, \frac{108}{A_{1}-108} \mapsto 0 \text { when } p_{g}(X) \mapsto+\infty,
$$

we can easily find a constant $K_{3}$ such that $g(C) \leq b_{0}+109$ provided $p_{g}(X) \geq$ $K_{3}$.

Lemma 2.6. Let $X$ be a Gorenstein minimal projective 3-fold of general type with at worst locally factorial terminal singularities. Suppose $\operatorname{dim} \phi_{1}(X)=2, \kappa(W)=$ $-\infty$ and $b_{0} \geq 3$. Then $p_{g}\left(S_{1}\right) \leq \frac{9}{2} K_{X}^{3}-2 p_{g}(X)+6$ where $S_{1}$ is by definition the movable part of $\left|K_{X^{\prime}}\right|$.

Proof. Because $W$ is birationally ruled, we have a projection map $p: W \longrightarrow B$ onto a smooth curve $B$ with genus $b_{0}$. Because $b_{0}>0, p$ is a morphism and a fibration. Because $\phi_{1}$ factors through $f$ and $\operatorname{dim} \phi_{1}(X)=2, \operatorname{dim} f\left(S_{1}\right)=1$. This means $f$ maps a general $S_{1}$ onto a curve $H$ in $W$ and $H$ is exactly the pull-back of a hyperplane section $H^{\prime}$ in $W^{\prime}$ under the map $s: W \longrightarrow W^{\prime}$. We see that $H$ is a nef and big divisor on $W$ and $H$ is a smooth irreducible curve. Therefore there is a finite map $\theta: H \longrightarrow B$, because $p$ doesn't contract $H$. So $b_{1}:=g(H) \geq g(B)=b_{0}$. We have the following commutative diagram:

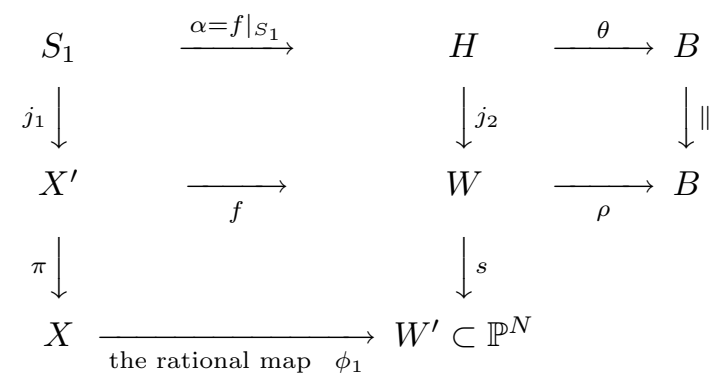

where $j_{1}$ and $j_{2}$ are both inclusion. We can see that $\alpha: S_{1} \longrightarrow H$ is a fibration where a general fiber $C^{\prime}$ lies in the same numerical class as a fiber of $f$. Thus $g\left(C^{\prime}\right)=g(C)$. Because $b_{0} \geq 3, q\left(S_{1}\right) \geq b_{1} \geq b_{0} \geq 3$. So $\left|K_{S_{1}}\right|$ defines a generically finite map onto its image according to [18]. Denote by $N_{1}$ the movable part of $\left|K_{S_{1}}\right|$. Then, according to Noether's inequality, $N_{1}^{2} \geq 2 p_{g}\left(S_{1}\right)-4$. We can bound 
$p_{g}\left(S_{1}\right)$ by studying $\phi_{3}$. It is obvious that $\left|K_{X^{\prime}}+\pi^{*}\left(K_{X}\right)+S_{1}\right| \subset\left|3 K_{X^{\prime}}\right|$. Denote by $M_{3}^{\prime}$ the movable part of $\left|K_{X^{\prime}}+\pi^{*}\left(K_{X}\right)+S_{1}\right|$ and by $M_{3}$ the movable part of $\left|3 K_{X^{\prime}}\right|$. Then we have $3 \pi^{*}\left(K_{X}\right) \geq M_{3} \geq M_{3}^{\prime}$. The vanishing theorem gives

$$
\left.\left|K_{X^{\prime}}+\pi^{*}\left(K_{X}\right)+S_{1}\right|\right|_{S_{1}}=\left|K_{S_{1}}+L\right| \supset\left|N_{1}+L_{0}\right|,
$$

where $L=\left.\pi^{*}\left(K_{X}\right)\right|_{S_{1}}$ and $L_{0}=\left.S_{1}\right|_{S_{1}}$. Because $S_{1}^{3}=0$, we can see that $\left|L_{0}\right|$ is composed of a free pencil of curves on the surface $S_{1}$. We can write $L_{0} \equiv a_{1} C^{\prime}$ where $a_{1} \geq p_{g}(X)-2$. Since $N_{1}+L_{0}$ is movable, we get from [5] (Lemma 2.7) that

$$
3 L \geq\left. M_{3}^{\prime}\right|_{S_{1}} \geq N_{1}+L_{0} .
$$

Thus $9 L^{2} \geq\left(N_{1}+L_{0}\right)^{2}$. Noting that $N_{1} \cdot C^{\prime} \geq 2$, we can easily derive the inequality

$$
p_{g}\left(S_{1}\right) \leq \frac{9}{2} K_{X}^{3}-2 p_{g}(X)+6 .
$$

Proposition 2.7. Let $X$ be a Gorenstein minimal projective 3 -fold of general type with at worst locally factorial terminal singularities. Suppose $\operatorname{dim} \phi_{1}(X)=2$, $\kappa(W)=-\infty$ and $b_{0} \geq 3$. If $g(C) \geq 648$, then $b_{0} \leq p_{g}(X)+653$.

Proof. According to the proof of Lemma 2.6 we have a surface fibration $\alpha: S_{1} \longrightarrow$ $H$ where $g\left(C^{\prime}\right)=g(C)$ and $b_{1}=g(H) \geq b_{0}$. We shall use a filtration of vector bundles on $H$ which was first studied by G. Xiao ([18]). Considering the natural map

$$
H^{0}\left(X^{\prime}, S_{1}\right) \stackrel{\gamma_{1}}{\longrightarrow} \Lambda_{1} \subset H^{0}\left(S_{1}, L_{0}\right)
$$

where $\Lambda_{1}$ is the image of $\gamma_{1}$, we can see that $\alpha$ is exactly obtained by taking the Stein factorization of $\Phi_{\Lambda_{1}, S_{1}}$. Note that $L_{0} \leq K_{S_{1}}$ and $H^{0}\left(S_{1}, \omega_{S_{1}}\right) \cong H^{0}\left(H, \alpha_{*} \omega_{S_{1}}\right)$ where $\alpha_{*} \omega_{S_{1}}$ is a vector bundle of rank $g(C)$ on the curve $H$. Denote by $\mathcal{L}_{0}$ the saturated sub-bundle of $\alpha_{*} \omega_{S_{1}}$, which is generated by sections in $\Lambda_{1}$. Then it is obvious that $\mathcal{O}_{L_{0}}$ is a line bundle because $\left|L_{0}\right|$ is composed of fibers of $\alpha$. So we get an extension of $\alpha_{*} \omega_{S_{1}}$ :

$$
0 \longrightarrow \mathcal{L}_{0} \longrightarrow \alpha_{*} \omega_{S_{1}} \longrightarrow \mathcal{L}_{1} \longrightarrow 0 .
$$

Because $\alpha_{*} \omega_{S_{1} / H}$ is semi-positive, we have $\operatorname{deg}\left(\mathcal{L}_{1} \otimes \omega_{H}^{-1}\right) \geq 0$, i.e., $\operatorname{deg}\left(\mathcal{L}_{1}\right) \geq$ $2(g(C)-1)\left(b_{1}-1\right)$. The Riemann-Roch theorem gives $h^{0}\left(H, \mathcal{L}_{1}\right) \geq(g(C)-1)\left(b_{1}-1\right)$. Noting that $\operatorname{deg}\left(\mathcal{L}_{0}\right)>0$, we get, by applying Clifford's theorem, that $h^{1}\left(H, \mathcal{L}_{0}\right) \leq$ $b_{1}-1$. From the long exact sequence

$$
0 \longrightarrow H^{0}\left(\mathcal{L}_{0}\right) \longrightarrow H^{0}\left(\alpha_{*} \omega_{S_{1}}\right) \longrightarrow H^{0}\left(\mathcal{L}_{1}\right) \longrightarrow H^{1}\left(\mathcal{L}_{0}\right) \longrightarrow \cdots,
$$

we have the inequality

$$
h^{0}\left(\mathcal{L}_{0}\right)-h^{0}\left(\alpha_{*} \omega_{S_{1}}\right)+h^{0}\left(\mathcal{L}_{1}\right)-h^{1}\left(\mathcal{L}_{0}\right) \leq 0 .
$$

Noting that $h^{0}\left(\mathcal{L}_{0}\right) \geq 2$ and using Lemma 2.6. we have

$$
2+(g(C)-2)\left(b_{1}-1\right) \leq p_{g}\left(S_{1}\right) \leq \frac{9}{2} K_{X}^{3}-2 p_{g}(X)+6 .
$$

Also noting that $b_{1} \geq b_{0}$ and using Miyaoka's inequality, one can get

$$
\begin{gathered}
(g(C)-2)\left(b_{0}-1\right) \leq 322 p_{g}(X)+324 b_{0}+324 g-320, \\
(g(C)-326) b_{0} \leq 322 p_{g}(X)+325 g-322 .
\end{gathered}
$$


If $g(C)>326$, we have

$$
b_{0} \leq \frac{322 p_{g}(X)}{g(C)-326}+325+\frac{105628}{g(C)-326} .
$$

It is easy to see $b_{0} \leq p_{g}(X)+653$ provided $g(C) \geq 648$.

Theorem 2.8. There exists a computable constant $K_{4}$ such that for all $X$, a Gorenstein minimal projective 3 -fold of general type with at worst locally factorial terminal singularities, satisfying $\operatorname{dim} \phi_{1}(X)=2$ and $\kappa(W)=-\infty$, we have $g(C) \leq 647$ provided $p_{g}(X) \geq K_{4}$.

Proof. If $b_{0} \leq 2$, then Corollary 2.5 gives $g(C) \leq 111$ provided $p_{g}(X) \geq K_{3}$. If $b_{0}>2$ and $g(C) \geq 648$, we have $b_{0} \leq p_{g}(X)+653$ by Proposition 2.7 Applying 2.2 and the Miyaoka-Yau inequality, we obtain

$$
\begin{aligned}
\frac{2}{3}(g(C)-1)\left[p_{g}(X)-2\right] & \leq 72\left[p_{g}(X)+b_{0}+g(C)-1\right] \\
& \leq 144 p_{g}(X)+72 g(C)+72 \cdot 652 .
\end{aligned}
$$

If $p_{g}(X)>110$, we can obtain

$$
g(C) \leq 217 \cdot \frac{p_{g}(X)}{p_{g}(X)-110}+\frac{70414}{p_{g}(X)-110} .
$$

One can easily find a constant $K_{4}^{\prime}$ such that $g(C) \leq 217$ provided $p_{g}(X) \geq K_{4}^{\prime}$. This contradicts the assumption $g(C) \geq 648$. Thus, when $p_{g}(X) \geq K_{4}:=\max \left\{K_{3}, K_{4}^{\prime}\right\}$, $g(C) \leq 647$.

Theorems 1.5, 2.3 and 2.8 imply Theorem 0.1

Example 2.9. We give a family of examples where $X$ is canonically fibred by curves of genus 3 and the canonical map is of constant moduli. The original idea comes from [1]. Let $W$ be a smooth projective surface with $p_{g}(W)=0$ and let $C_{0}$ be a smooth curve of genus 2. Denote $X_{0}:=W \times C_{0} . p_{1}: X_{0} \longrightarrow W$ and $p_{2}: X_{0} \longrightarrow C_{0}$ are projections. Suppose we have a divisor $H$ on $W$ such that $|2 H|$ is base point free, $h^{0}(W, 2 H) \geq 2$ and that $\left|K_{W}+H\right|$ gives a generically finite map. Let $\theta=P-Q$ be a divisor on $C_{0}$ such that $2 \theta \sim 0$. On $X_{0}$, denote $\delta:=p_{1}^{*}(H)+p_{2}^{*}(\theta)$. Then we can see that $\delta$ determines a smooth double cover $\pi: X \longrightarrow X_{0}$ with

$$
\begin{gathered}
K_{X}=\pi^{*}\left(K_{X_{0}}+\delta\right), \\
p_{g}(X)=h^{0}\left(X_{0}, p_{1}^{*}\left(K_{W}+H\right)\right) .
\end{gathered}
$$

Because $h^{0}\left(C_{0}, K_{C_{0}}+\theta\right)=1$, we can see that $\Phi_{K_{X}}$ factors through $\pi$ and $p_{1}$. If $K_{W}+H$ is nef, then $X$ is minimal. Denote $f:=p_{1} \circ \pi$. Then $f$ is the derived fibration from $\phi_{1}$ and a general fiber of $f$ is a curve of genus 3 .

2.10. Open problem. It is quite interesting to consider a parallel problem. Let $X$ be a Gorenstein minimal projective 3 -fold with at worst locally factorial terminal singularities. Suppose $\phi_{1}$ is generically finite onto its image. Is the generic degree of $\phi_{1}$ universally upper bounded? We don't know any work on this problem. It is well known that the upper bound is 16 in the surface case. 


\section{REFERENCES}

[1] A. Beauville, L'application canonique pour les surfaces de type général, Invent. Math. 55(1979), 121-140. MR0553705 (81m:14025)

[2] _ L'inégalité $p_{g} \geq 2 q-4$ pour les surfaces de type général, Bull. Soc. Math. France 110(1982), 344-346. MR0688038 (84f:14026)

[3] E. Bombieri, Canonical models of surfaces of general type, Publications I.H.E.S. 42(1973), 171-219. MF $0318163(47: 6710)$

[4] M. Chen, Complex varieties of general type whose canonical systems are composed with pencils, J. Math. Soc. Japan 51(1999), 331-335. MR1674752 (2000b:14039)

[5] _ Canonical stability in terms of singularity index for algebraic threefolds, Math. Proc. Camb. Phil. Soc. 131(2001), 241-264. MR,1857118 (2002i:14041)

[6] Inequalities of Noether type for 3-folds of general type, J. Math. Soc. Japan 56 (2004), 1131-1155.

[7] M. Chen, Z. Chen, Irregularity of canonical pencils for a threefold of general type, Math. Proc. Camb. Phil. Soc. 125(1999), 83-87. MR1645525 (99g:14047)

[8] T. Fujita, On Kahler fiber spaces over curves, J. Math. Soc. Japan 30 (1978), 779-794. MR 0513085 (82h:32024)

[9] H.Hironaka, Resolution of singularities of an algebraic variety over a field of characteristic zero, I, Ann. of Math. 79(1964), 109-203, II, ibid., 205-326. MR0199184 (33:7333)

[10] Y. Kawamata, A generalization of Kodaira-Ramanujam's vanishing theorem, Math. Ann. 261(1982), 43-46. MR0675204 (84i:14022)

[11] Y. Kawamata, K. Matsuda, K. Matsuki, Introduction to the minimal model problem, Adv. Stud. Pure Math. 10(1987), 283-360. MR0946243 (89e:14015)

[12] J. Kollár, Higher direct images of dualizing sheaves, I, Ann. of Math. 123(1986), 11-42; II, ibid. 124(1986), 171-202. MR0825838 (87c:14038) MR0847955|(87k:14014)

[13] J. Kollár, S. Mori, Birational geometry of algebraic varieties, 1998, Cambridge Univ. Press. MR.1658959 (2000b:14018)

[14] Y. Miyaoka, The pseudo-effectivity of $3 c_{2}-c_{1}^{2}$ for varieties with numerically effective canonical classes, Algebraic Geometry, Sendai, 1985. Adv. Stud. Pure Math. 10(1987), 449-476.

[15] E. Viehweg, Vanishing theorems, J. reine angew. Math. 335(1982), 1-8. MR0667459 (83m:14011)

[16] Weak positivity and the additivity of the Kodaira dimension for certain fibre spaces, Adv. Stud. Pure Math. 1(1983), 329-353. MR0715656 (85b:14041)

[17] _ Weak positivity and the additivity of the Kodaira dimension, II: The local Torelli map, Classification of algebraic and analytic manifolds, Prog. Math. 39(1983), 567-589. MR 0728619 (85i:14020)

[18] G. Xiao, L'irrégularité des surfaces de type général dont le système canonique est composé d'un pinceau, Comp. Math. 56(1985), 251-257. MR0809870 (87d:14031)

Institute of Mathematics, Fudan University, Shanghai, 200433, People's Republic of China

E-mail address: mchen@fudan.edu.cn 